\title{
Coherent diffractive imaging of single helium nanodroplets with a high harmonic generation source
}

\author{
Daniela Rupp¹, Nils Monserud², Bruno Langbehn', Mario Sauppe1, Julian Zimmermann (1) 1, \\ Yevheniy Ovcharenko1,3, Thomas Möller ${ }^{1}$, Fabio Frassetto ${ }^{4}$, Luca Poletto ${ }^{4}$, Andrea Trabattoni ${ }^{4,5}$, \\ Francesca Calegari ${ }^{5,6}$, Mauro Nisoli ${ }^{6,7}$, Katharina Sander ${ }^{8}$, Christian Peltz $^{8}$, Marc J. Vrakking ${ }^{2}$, \\ Thomas Fennel (1D ${ }^{2,8}$ \& Arnaud Rouzée ${ }^{2}$
}

Coherent diffractive imaging of individual free nanoparticles has opened routes for the in situ analysis of their transient structural, optical, and electronic properties. So far, single-shot single-particle diffraction was assumed to be feasible only at extreme ultraviolet and X-ray free-electron lasers, restricting this research field to large-scale facilities. Here we demonstrate single-shot imaging of isolated helium nanodroplets using extreme ultraviolet pulses from a femtosecond-laser-driven high harmonic source. We obtain bright wide-angle scattering patterns, that allow us to uniquely identify hitherto unresolved prolate shapes of superfluid helium droplets. Our results mark the advent of single-shot gas-phase nanoscopy with lab-based short-wavelength pulses and pave the way to ultrafast coherent diffractive imaging with phase-controlled multicolor fields and attosecond pulses.

\footnotetext{
${ }^{1}$ Institut für Optik und Atomare Physik, Technische Universität Berlin, Hardenbergstraße 36, 10623 Berlin, Germany. ${ }^{2}$ Max-Born-Institut für Nichtlineare Optik und Kurzzeitspektroskopie, Max-Born-Straße 2A, 12489 Berlin, Germany. ${ }^{3}$ European XFEL GmbH, Holzkoppel 4, 22869 Schenefeld, Hamburg, Germany. ${ }^{4}$ CNR, Istituto di Fotonica e Nanotecnologie Padova, Via Trasea 7, 35131 Padova, Italy. ${ }^{5}$ Center for Free-Electron Laser Science, DESY, Notkestr. 85, 22607 Hamburg, Germany. ${ }^{6}$ CNR, Istituto di Fotonica e Nanotecnologie Milano, Piazza L. da Vinci 32, 20133 Milano, Italy. ${ }^{7}$ Department of Physics, Politecnico di Milano, Piazza L. da Vinci 32, 20133 Milano, Italy. ${ }^{8}$ Institut für Physik, Universität Rostock, Albert-Einstein-Straße 23, 18059 Rostock, Germany. Correspondence and requests for materials should be addressed to D.R. (email: daniela.rupp@physik.tu-berlin.de) or to

T.F. (email: thomas.fennel@uni-rostock.de) or to A.Rée. (email: arnaud.rouzee@mbi-berlin.de)
} 
S ingle-shot coherent diffractive imaging (CDI) with intense short-wavelength pulses became possible just recently with the advent of extreme ultraviolet (XUV) and X-ray freeelectron lasers $(\mathrm{FEL})^{1}$. This lensless imaging method has revolutionized the structural characterization of nanoscale samples, including biological specimens ${ }^{2}$, aerosols ${ }^{3}$, atomic clusters $^{4-6}$, and nanocrystals ${ }^{7}$. By capturing high-quality diffraction patterns from a single nanoparticle in free flight using a single laser pulse, the sample morphology can be determined in situ and free from spurious interactions due to deposition on a substrate. For sufficiently regular structures the wide-angle scattering information even reveals the full three-dimensional particle shape and orientation ${ }^{6-8}$, as multiple projections of the same particle are encoded in a single diffraction image ${ }^{6}$. These unique capabilities enable the investigation of metastable or transient states that exist only in the gas phase. Pioneering FEL experiments have explored this frontier and demonstrated CDI of quantum vortices in helium droplets ${ }^{5}$, ultrafast nanoplasma formation ${ }^{9}$, and explosion of laser-heated clusters ${ }^{10}$. Using XUV and soft X-ray high harmonic generation (HHG) sources for single-shot nanoparticle CDI holds the promise to combine the nanoscale structural imaging capabilities of CDI with the exquisite temporal, spectral, and phase control inherent in the use of optical lasers, including the fascinating prospect of CDI with isolated attosecond pulses.

The brightness of HHG sources is typically orders of magnitude lower than that of an FEL ${ }^{11}$, but over the years, a number of them have been scaled up in order to achieve high intensities and/ or high average power ${ }^{12-17}$, allowing CDI of fixed targets ${ }^{18-23}$. Experiments on ion-beam-edged nanostructures in membranes achieved impressive resolution on the order of $20 \mathrm{~nm}$ after multiple exposures 22,23 and even yielded the overall shape from a single-shot diffraction image in favorable cases ${ }^{20}$. Here we report the feasibility of lab-based $3 \mathrm{D}$ characterization of unsupported nanoparticles and demonstrate single-shot HHG-CDI on individual free helium nanodroplets.

\section{Results}

Single-shot scattering with a high harmonic source. In our experiment (Fig. 1), a high power Ti:sapphire laser amplifier was used to generate $35 \mathrm{fs}$ laser pulses at $792 \mathrm{~nm}$ wavelength with up to $33 \mathrm{~mJ}$ pulse energy. About $12 \mathrm{~mJ}$ were loosely focused $(f=5 \mathrm{~m})$ into a xenon-filled gas cell ${ }^{24}$, producing $\approx 2 \mu \mathrm{J}$ of XUV radiation, i.e., close to $10^{12}$ photons per pulse. This corresponds to $\sim 1 \%$ of the pulse energy that can currently be achieved at the XUV freeelectron laser FERMI ${ }^{25}$. The CDI application requires high fluence, and thus tight focusing. Typical back-reflection multilayer mirrors, however, conflict with the use of straylight apertures and the detection of scattered light at small scattering angles. Therefore, CDI-compatible grazing-incidence microfocusing optics with an overall transmission of $10 \%$, based on a coma-correcting system of toroidal mirrors ${ }^{26}$, were used to focus the multicolor XUV beam to a small spot (beam-waist $\omega_{0}=10 \mu \mathrm{m}$ ), achieving a power density of $3 \times 10^{12} \mathrm{~W} \mathrm{~cm}^{-2}$ (pulse averaged). The XUV spectrum (11th to 17 th harmonic, see Fig. $2 \mathrm{~d}$ ) was obtained prior to the CDI measurements with a grating spectrometer. A jet of helium droplets with diameters of several hundreds of nanometers crossed the focus of the XUV beam. The droplets were generated using a cryogenically cooled pulsed valve maintained at a temperature between 4.9 and $5.7 \mathrm{~K}$, operating at low repetition rates of $3-10 \mathrm{~Hz}$. The diffracted radiation was measured shot-toshot with a wide-area MCP (micro-channel plate)-based scattering detector (see "Methods" section).

Analysis of the multicolor diffraction patterns. Within $3 \times 10^{5}$ single-shot measurements, 2300 bright patterns with distinct structures were obtained and another 12,700 recorded images contained weak, unstructured scattering signal. A selection of exemplary diffraction patterns is displayed in Fig. 3. To analyze the diffraction patterns, the multiple spectral components of the HHG pulses have to be taken into account. The simultaneous use of several different wavelengths complicates the analysis of the size and shape of the particles, but it is also a fundamental precondition for generating attosecond pulse trains and isolated attosecond pulses ${ }^{27}$. Therefore, all future approaches toward attosecond diffractive imaging will require a multicolor analysis. We developed a multidimensional Simplex optimization ${ }^{28}$ on the basis of multicolor Mie scattering calculations ${ }^{29,30}$ to analyze the diffraction images of spherical helium droplets and to demonstrate that the fits can give access to the optical properties of the droplets.

The majority of bright diffraction patterns $(\approx 76 \%)$ shows ring structures that can be assigned to spherical droplets (cf. Fig. 2a). The diffracted field from dielectric spheres illuminated with a single wavelength can be described by the Mie solution and yields scattering patterns showing concentric rings ${ }^{29}, 30$. The ring separation scales with the wavelength and with the inverse particle size. The overall scattering strength and the detailed shape of the ring structure further depend on the material's complex refractive index (see "Methods" section). In our case, the XUV pulses contain spectral contributions from four harmonics (Fig. 2d). Therefore, the scattering pattern is a superposition of the corresponding single-wavelength scattering intensities, and displays a characteristic beating pattern due to the wavelengthdependent ring spacings of the individual spectral contributions to the image (black curve in Fig. 2b, c). The observed patterns are fitted via a multidimensional Mie-based optimization with the

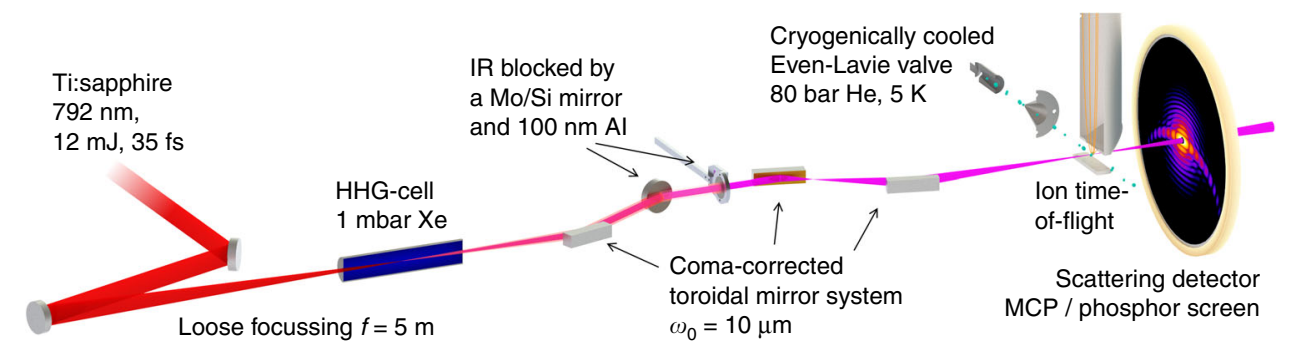

Fig. 1 Scheme of the experimental setup. A Ti:sapphire laser with $792 \mathrm{~nm}$ central wavelength and $35 \mathrm{fs}$ pulse duration is used for the generation of high harmonics. Up to $12 \mathrm{~mJ}$ are loosely focused into a xenon-filled cell, where the extreme ultraviolet (XUV) pulses are produced. The copropagating nearinfrared (NIR) beam is removed via a Mo/Si mirror and a thin aluminum filter. The beam is focused to a small spot ( $\left.\omega_{0}=10 \mu \mathrm{m}\right)$ using a coma-correcting system of three gold-coated toroidal mirrors ${ }^{26}$. A pulsed jet of helium nanodroplets $(\bar{R} \approx 400 \mathrm{~nm})$ is overlapped with the $\mathrm{XUV}$ focus. The overlap is optimized by monitoring the formation of $\mathrm{He}^{+}$ions using an ion time-of-flight spectrometer. The scattering signal is amplified by a pulsed $\mathrm{MCP}$ and converted to optical photons on a phosphor screen. The single-shot diffraction images are captured with an out-of-vacuum camera (not depicted) 

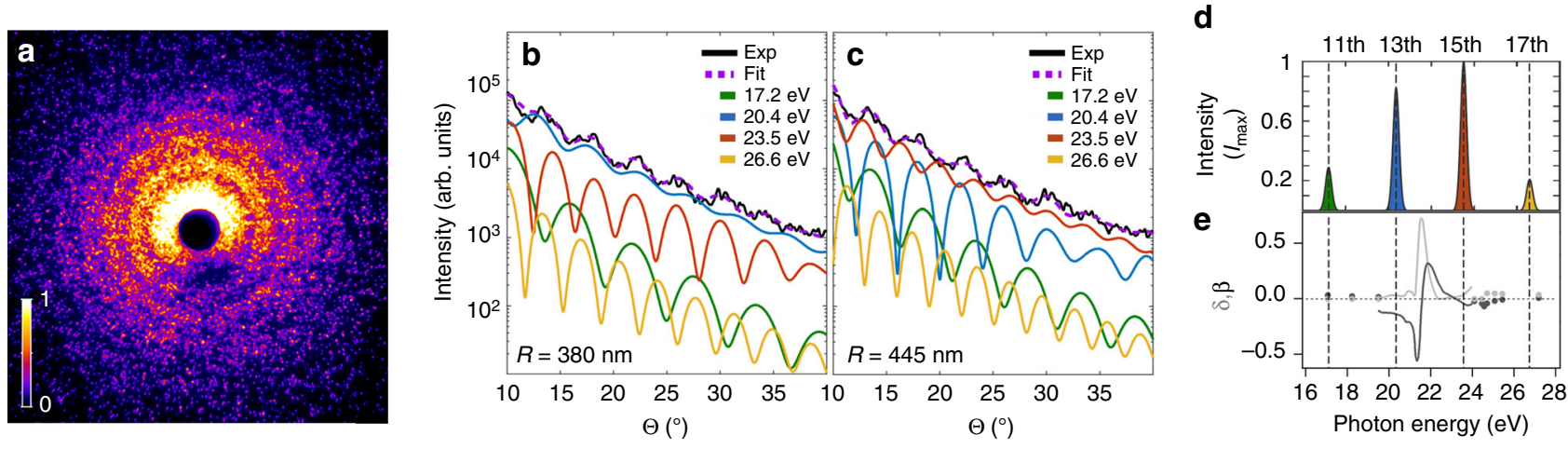

Fig. 2 Multicolor analysis of the diffraction images. a Measured bright scattering image (center part of the detector, intensity in arbitrary units) from a spherical droplet with a pronounced concentric ring pattern. b, c Multicolor Mie fits (dashed purple) of the extracted radial intensity profile (solid black) from a as obtained via a simplex optimization (see "Methods" section) of the individual harmonic contributions to the profiles (color-coded in green, blue, red, and yellow). The results illustrate that two qualitatively different solutions yield comparably small residuals. The two solutions indicate that either the 13 th

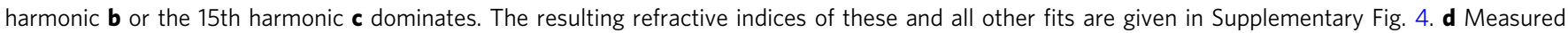
average extreme ultraviolet spectrum of the high harmonic radiation. e Sketch of the energy-dependent refractive indices of bulk liquid helium in the vicinity of the helium 1s-2p transition, assembled from bulk liquid helium measurements ${ }^{31,} 32$ (solid lines, color-coded in light-gray and dark-gray) and tabulated values (scatter, NIST database, http://physics.nist.gov/PhysRefData/FFast/html/form.html)

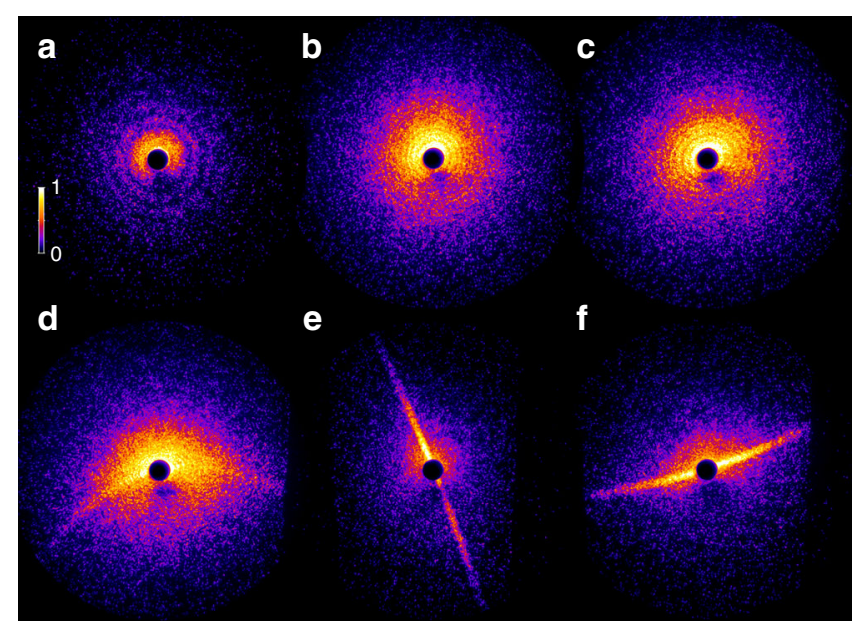

Fig. 3 Three characteristic types of diffraction images from helium nanodroplets. The majority of images contains concentric ring patterns $\mathbf{a}, \mathbf{b}$ that are assigned to spherical droplets. Elliptical ring structures as in c or pronounced streak patterns as in $\mathbf{d - f}$ reflect deformed helium droplets. The measured dataset comprises 1762 ring-type, 421 elliptical, and 68 streaktype images. In most cases, the latter exhibit a clear bending of the streaks (55 out of 68 images), e.g., as in $\mathbf{d}$ and $\mathbf{f}$. For details, see Supplementary Note 3. False color images, color bar applies to all patterns, intensity in arbitrary units

particle size, the refractive indices at the wavelengths of the contributing harmonics, the relative intensities of the harmonics, and a scaling factor for the total intensity of the XUV pulse as input parameters (see "Methods" section). While the optical properties of bulk liquid helium have been measured and calculated close to the $1 s-2 p$ transition of helium ${ }^{31,32}$ (see Fig. 2e), the dielectric function of the nanodroplets is completely unknown and expected to vary substantially with droplet size ${ }^{33}$. In fact, we find that fits using the bulk literature values for the refractive indices at the corresponding harmonic wavelengths cannot reproduce the observed diffraction patterns (see also Supplementary Note 1).

Successful fits can be achieved by using the refractive indices at the wavelengths of the dominant 13th and 15th harmonics as optimization parameters in addition to the particle size. In this procedure, the relative intensities of the contributing harmonics are set to measured average values and the refractive indices at the wavelengths of the 11th and 17th harmonics are fixed, as they lie far away from the large helium resonances (cf. Fig. 2e). The optimization was successfully carried out for 18 very bright scattering patterns with clear beating structures up to large scattering angles. The majority of these fits indicate a dominant contribution of the 13th harmonic, as exemplified in Fig. 2b. However, for all patterns, a second solution with dominant signal from the 15th harmonic is found by the algorithm with comparably low residuals of the fit (cf. Fig. $2 \mathrm{c}$ and Supplementary Note 2). As the similarly intense 13th and 15th harmonics lie close to each other (only $3.1 \mathrm{eV}$ energetic distance), the fitting algorithm can vary the refractive indices freely and exchange their roles in the fit, while adjusting the cluster size accordingly (compare Fig. 2b, c). We note that while the residuals of the fits are slightly smaller for the solution with dominant 13th harmonic, the refractive indices for the solution with dominant 15th harmonic lie closer to the literature values of bulk helium. However, we cannot fully exclude one of the two solutions. In order to resolve such ambiguity in the optimization, future systematic studies are required with only one of the strong harmonics being near-resonance and/or with substantially better signal to noise ratio. By using higher energy and/or lower wavelength lasers to drive the HHG process, it is anticipated that the photon flux of individual harmonics can be further increased by at least one order of magnitude ${ }^{17}$, while the energetic distance between the harmonics becomes larger. However, the present analysis supports that the multicolor fit procedure can be used for a new metrology of optical parameters and constitutes a basis toward future multicolor imaging approaches. In the subsequent scattering simulations, the average values of the refractive indicies from the solutions with dominant 13th harmonic are used (see "Methods" section).

Identification of prolate droplet shapes. Besides concentric ring patterns (Fig. 3a, b) and an abundance of about $20 \%$ of elliptical patterns from ellipsoidal droplets (cf. Fig. 3c), about 3\% of all bright images exhibit pronounced streak structures as exemplified in Fig. $3 \mathrm{~d}-\mathrm{f}$ and Fig. 4a. The abundances of the three main types of patterns, i.e., rings, elliptical and streak patterns, are similar to what has been reported in previous hard X-ray measurements at 


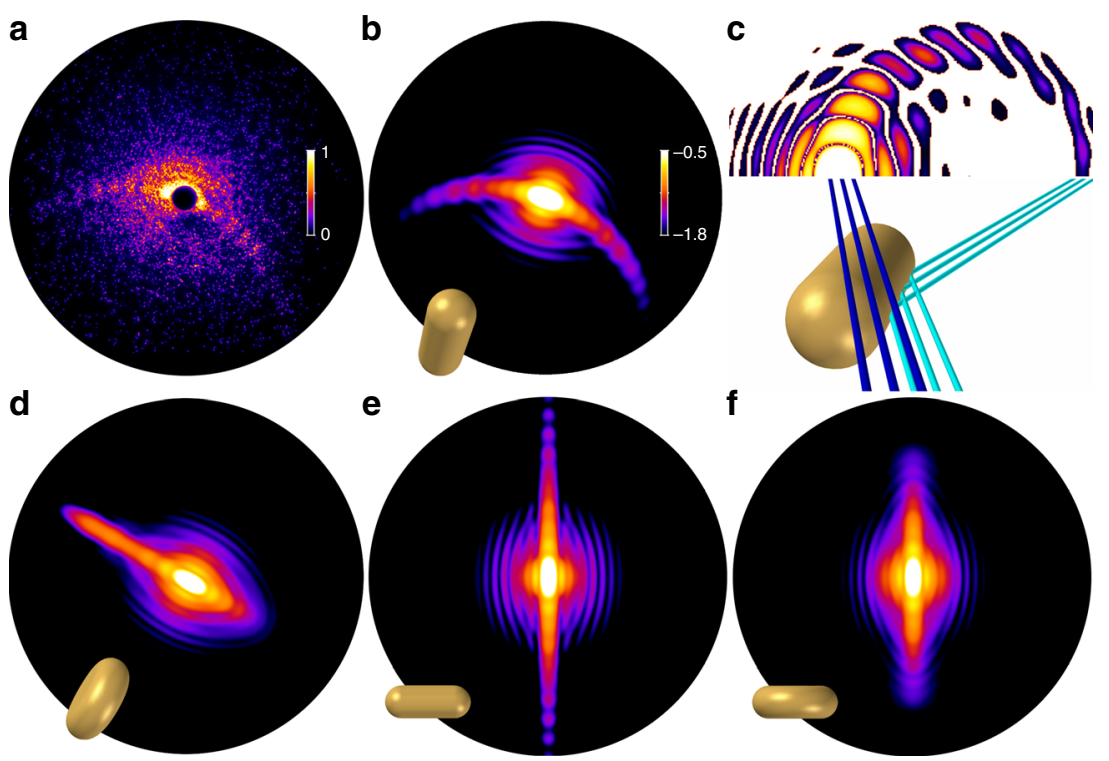

Fig. 4 Unique identification of prolate pill-shaped structures. a Measured image (intensity in arbitrary units) and $\mathbf{b}$ matching simulation result of the wide-angle diffraction of a pill-shaped prolate droplet (logarithmic intensity in arbitrary units, color bar applies also to $\mathbf{d}-\mathbf{f}$ ). The structure shape and orientation are visualized in yellow. The optical axis of the extreme ultraviolet beam is directed into the image plane, the tilt angle between the symmetry axis of the particle and the optical axis is $35^{\circ}$; the semi-minor axes $a=b=370 \mathrm{~nm}$ and the semi-major axis $c=950 \mathrm{~nm}$; for optical parameters see "Methods" section. c Illustration of the origin of bent streaks occurring when a tilted rod-type structure diffracts the light. The constructive interference is analogous to the specular reflection at the surface of a macroscopic rod. Two particular bundles of constructively interfering rays are explicitly sketched, please note that the different ray colors do not refer to wavelengths, but are applied to facilitate distinction. $\mathbf{d}$ Simulated wide-angle diffraction image of a wheel-shaped oblate particle (semi-major/-minor axes as in $\mathbf{b}$, tilt angle of $80^{\circ}$ between the symmetry axis and the optical axis, which is directed into the image plane). If the oblate particle's symmetry axis is neither oriented along the optical axis nor perpendicular to it, the diffraction patterns exhibit straight streaks to only one side. e, $\mathbf{f}$ Comparison of simulated wide-angle diffraction images of a prolate $\mathbf{e}$ and an oblate structure $\mathbf{f}$ aligned to the scattering plane, i.e., at $90^{\circ}$ tilt angle between the symmetry axis and the optical axis, other parameters as in $\mathbf{b}$, $\mathbf{d}$. Though the 2D projections are similar and the 2D outlines identical, the intensity distributions of the straight streaks are clearly different and decay much faster for wheel-type than for pill-type shapes

LCLS $^{5}$. However, whereas straight streak patterns were observed in the X-ray results, we find in the majority of our wide-angle scattering patterns a pronounced crescent-shaped bending of the streaks (statistics see Fig. 3 and Supplementary Note 3). In the Xray experiments, the reconstruction of the corresponding droplet shapes via iterative phase retrieval revealed $2 \mathrm{D}$ projections of the helium droplets with extreme aspect ratios ${ }^{5}$. These were assigned to extremely flattened, wheel-like oblate shapes. The deformation was attributed to a high angular momentum, which can be transferred to the droplets by cavitation and rip-off from the liquid phase during the formation process ${ }^{5,34}$, while vibrational excitations are assumed to decay very quickly ${ }^{35}$. Whereas classical viscid rotating droplets undergo a deformation from oblate to prolate two-lobed shapes with the rotation axis perpendicular to the long axis of the droplett ${ }^{36,37}$, this transition has been suggested to be hindered in helium nanodroplets by the appearance of vortex arrays that deform the droplets and stabilize extreme oblate shapes ${ }^{38}$. Very recently, the occurence of such classically unstable oblate helium droplets was further supported by statistical arguments, while an indication of rare prolate structures was also found ${ }^{39}$. However, a unique discrimination of prolate and oblate shapes based on the $2 \mathrm{D}$ projections accessible with smallangle X-ray diffraction is difficult ${ }^{39}$. Tomographic information, on the other hand, is contained in XUV wide-angle scattering and can be exploited to retrieve the three-dimensional particle shape and orientation provided the particle morphology is sufficiently regular ${ }^{6,7}$. In our measurement, the presence of tomographic information prominently manifests in the bending of the streak patterns.

In order to retrieve the shapes underlying the experimentally observed scattering patterns, three-dimensional multicolor scattering simulations were performed ${ }^{40}$ (see "Methods" section). The two-sided, bent streak features can only be reproduced, considering prolate droplets as shown in Fig. $4 \mathrm{~b}$, matching the experimental pattern of Fig. 4a. Crescent-shaped streaks arise from prolate structures that are tilted out of the scattering plane (i.e., the plane normal to the laser propagation axis). The wideangle interference pattern can be intuitively understood in analogy to the reflection of a laser from a macroscopic rod. As shown in Fig. 4c, bundles of rays diffracted by the cylindrical part of the surface gather the same path length and interfere constructively. In contrast, a tilted wheel-shaped particle with the same aspect ratio as exemplified in Fig. $4 \mathrm{~d}$ cannot explain the bending of the streaks. Instead, in the wide-angle scattering regime, the simulations indicate that such particles would generate a one-sided straight streak, a phenomenon that was not observed in our experiment. Although less obvious, also the analysis of observed straight streak patterns indicates that they can only be explained by prolate structures, as the observed streak signal is visible until the edge of the detector (cf. for example Fig. 3e). In figure 4, panels e and $\mathrm{f}$ show a comparison of diffraction patterns for pill- and wheel-shaped structures, that are aligned to the scattering plane. The tomographic nature of wideangle scattering reveals that the streaks decay much faster toward larger scattering angles for a wheel than for a pill-shaped particle. We would like to note that the absence of wheel-shaped droplets in our experiment may stem from the different droplet generation scheme, using a pulsed valve with a long nozzle in this work, where the transition to the superfluid state might be delayed compared to the short CW flow nozzle used by Gomez et al. ${ }^{5}$. However, our unambiguous observation of prolate droplets, which are known to occur for classical liquids 36,37 , 
will contribute to the discussion on the stability of spinning superfluid droplets. Their existence may provide a fascinating case for future experiments, as it should be possible to clarify if a prolate droplet shows macroscopic-shape rotation, which is not expected for a superfluid droplet ${ }^{38}$.

\section{Discussion}

We have shown the feasibility of single-shot single-particle CDI using intense XUV pulses from a HHG source. Bright diffraction patterns of spherical helium droplets have been obtained and matched with simulations using optimized refractive indices. The observed crescent-shaped streak patterns could be uniquely assigned to prolate droplets. The results further suggest several future prospects connected to the HHG-specific properties. Laser-based HHG provides a high accessibility compared to FEL facilities in a wavelength regime suitable for $3 \mathrm{D}$ shape characterization of non-reproducible gas-phase nano-objects, particularly if experiments with single harmonics can be realized, which is anticipated using UV or deep UV driver lasers for $\mathrm{HHG}^{17},{ }^{41}$. This will facilitate fundamental investigations of structure formation, such as tracing ice nucleation ${ }^{42}$, with important implications for atmospheric physics and aerosol science. Moreover, unprecedented experiments beyond structural determination are possible, such as multicolor tomography and resonant-pump-resonant-probe $\mathrm{CDI}$, which exploit the time-resolution and phase control achievable in HHG-based experiments for diffractive imaging of quantum coherent dynamics. The spatiotemporal characterization of ultrafast electron dynamics has been driving attosecond science ${ }^{27,43}$ from the beginning and will perhaps be the most exciting prospect of HHG-CDI. Considering the advancing capability of generating intense isolated attosecond pulses ${ }^{15}$ and the possibility of stroboscopic illumination using attosecond pulse trains ${ }^{44}$, the vision of diffractive imaging of attosecond electron dynamics in isolated nanostructures has come in reach.

\section{Methods}

Femtosecond laser system and generation of XUV harmonics. The experiments are performed using a commercially available cryo-cooled Ti:sapphire laser amplifier (KMLabs Red Wyvern) delivering pulses at a central wavelength of 792 $\mathrm{nm}$ with $33 \mathrm{~mJ}$ pulse energy and $35 \mathrm{fs}$ pulse duration at $1 \mathrm{kHz}$ repetition rate. A fraction of $30 \%$, of the output energy (typically $10-12 \mathrm{~mJ}$ ), are taken for the generation of high harmonics. To this aim, a broadband spherical mirror with a focal distance of $5 \mathrm{~m}$ is used to focus the near-infrared (NIR) pulses into a $100 \mathrm{~mm}$ long aluminum gas cell statically filled with $\approx 1.3$ mbar of xenon (loose focusing geometry). The position of the gas cell, the gas pressure and the NIR pulse energy are adjusted to optimize the HHG flux. An output energy of $\sim 2 \mu$ (measured with a calibrated photodiode) is achieved in this geometry, corresponding to a conversion efficiency of $1.6 \times 10^{-4}$ and an average power of $2 \mathrm{~mW}$. To the best of our knowledge, this is the highest average power obtained by means of HHG. The harmonic beam consists of the 11th $(72 \mathrm{~nm}), 13$ th $(61 \mathrm{~nm}), 15$ th $(53 \mathrm{~nm})$, and 17th $(47 \mathrm{~nm})$ harmonics (see Fig. $2 \mathrm{~d}$ ) as measured by dispersing the XUV beam with a grating spectrometer. The XUV pulse duration was characterized in previous experiments using $\mathrm{THz}$ electron streaking technique to be roughly $20 \mathrm{fs}$.

Microfocusing setup and IR filter. A high throughput XUV beamline (transmission $\approx 10 \%)$ consisting of three gold-coated, grazing incidence $\left(10^{\circ}\right)$ toroidal mirrors and a flat $\mathrm{Mo} / \mathrm{Si}$ mirror is used to tightly focus the XUV beam in the interaction region. Positions, radii and distances between the toroidal mirrors were optimized by ray tracing in order to achieve a high demagnification factor of 25 for the XUV beam while keeping the coma-aberrations low ${ }^{26,45}$. The first $40 \mathrm{~mm} \times 10$ $\mathrm{mm}$ toroidal mirror with radii $57.6 \mathrm{~m} \times 1.735 \mathrm{~m}$ is placed $5 \mathrm{~m}$ away from the gas cell in order to collimate the XUV beam. The collimated beam is then reflected by a flat Mo/Si mirror that partially absorbs the co-propagating NIR laser pulse used for HHG. The remaining NIR laser beam $(\approx 1 \mathrm{~mJ})$ is filtered out by a $100 \mathrm{~nm}$ thin aluminum filter. We note that the reduction of the NIR by the Mo/Si mirror is required to avoid damaging the aluminum foil. A coma-corrected system composed of two toroidal mirrors facing each other is then used to demagnify and tightly focus the XUV beam into the experimental chamber. The first of the latter two toroidal mirrors (radii $2650 \mathrm{~mm} \times 79 \mathrm{~mm}$ ) has a focal length of $230 \mathrm{~mm}$. Subsequently, the last toroidal mirror (radii $3620 \mathrm{~mm} \times 109.2 \mathrm{~mm}$ ) is placed 680 $\mathrm{mm}$ away from the focus to relay image the focus of the first toroidal mirror into the experimental chamber at a distance of $585 \mathrm{~mm}$. This geometry allows a demagnification factor of 25 with respect to the initial XUV spot size at the generation point. Considering a $0.5 \mathrm{mrad} \times 0.5 \mathrm{mrad}$ diverging XUV beam with a 175 $\mu \mathrm{m}$ spotsize (FWHM) at the source point, we expect to achieve a minimum spot size at the focus of $7 \mu \mathrm{m}$ (FWHM). In our experiment, the size of the XUV beam was characterized by monitoring the fluorescence of a $\mathrm{Cs} / \mathrm{YAG}$ screen placed at the focus using a CCD camera. We measured a $9 \mu \mathrm{m} \times 10 \mu \mathrm{m}$ spot size (FWHM) with a $10 \mu \mathrm{m}$ beam waist, in close agreement with the expected value, leading to an intensity of $I_{\mathrm{f}}=3 \times 10^{12} \mathrm{~W} \mathrm{~cm}^{-2}$.

Helium droplet generation. The helium nanodroplets are generated with a pulsed Even-Lavie valve ${ }^{46}$ that is cooled with a Sumitomo closed-cycle cryostat down to 4.9-5.7 K. The minimum temperature depends on the repetition rate and the opening duration of the valve (varied between 3 and $10 \mathrm{~Hz}$ and 18 to $27 \mu$ s, respectively), which influence the heat load of the valve. High-purity ${ }^{4} \mathrm{He}$ (99.9999\%) at a pressure of 80 bars is expanded into a differentially pumped UHV chamber through a $100 \mu \mathrm{m}$ trumpet-shaped nozzle located at $450 \mathrm{~mm}$ distance to the interaction region. The droplet pulse is guided into the interaction chamber through a conical skimmer with $1 \mathrm{~mm}$ diameter, which reduces the uncondensed gas in the interaction chamber.

Scattering experiment. A large-area scattering detector (Ø: $75 \mathrm{~mm})$ with a center hole $(\varnothing: 3 \mathrm{~mm})$ is placed $37 \mathrm{~mm}$ behind the XUV focus, corresponding to a maximum spatial frequency of $0.09 \mathrm{~nm}^{-1}$ for the dominant wavelength of $53 \mathrm{~nm}$. The detector consists of a Chevron-type MCP for signal amplification and a phosphor screen for conversion to optical light ${ }^{4}$. The MCP is used in pulsed operation to suppress background signal from charged particles. Further, the CDI-compatible focusing geometry described above allows for the use of two straylight apertures before the focus to minimize photonic background signal on the detector. The $8^{\circ}$ tilt of the MCP channels results in an area with decreased response observable at the lower right side of the detector hole (cf. Figs. $2 \mathrm{a}, 3$ and $4 \mathrm{a})^{47}$. The scattering patterns are recorded on a shot-to-shot basis using an out-ofvacuum CMOS camera. An ion time-of-flight spectrometer is used for establishing and optimizing the spatial overlap of the XUV pulses and the helium nanodroplets and the timing of the droplet jet ${ }^{48}$. Within $3 \times 10^{5}$ single-shot measurements 2300 bright patterns with distinct structures were obtained. Further 12,700 recorded images contained weak, unstructured scattering signal. These statistics indicate that the experiment is performed in the single-particle limit as the probability to have two droplets in the focus at the same time is $<2 \%$.

Data analysis and scattering simulations. For comparison with theory, the measured diffraction patterns were transformed to the scattered intensity that would be recorded on a spherical detector. In addition, the measured data must be corrected for the nonlinear detection efficiency of the $\mathrm{MCP}^{6,9}$. Previous work has shown that the saturation effect can be described by an exponential efficiency function ${ }^{6}$ such that the detected signal intensity, $I_{\mathrm{det}}$, is connected to the true experimental intensity, $I_{\exp }$, via $I_{\text {det }}=I_{\text {exp }}^{\alpha}$. The nonlinearity exponent $\alpha=0.5$ has been found by matching the angular decay of the envelope of scattering profiles from spherical droplets to the universal $q^{-4}$ decay behavior predicted by Porod's $\operatorname{law}^{49}, 50$. The center position was independently determined for every pattern to correct for slight variations resulting from wavefront tilts at the position of the drople $^{51}$. Radial intensity profiles were extracted by angular averaging over the upper half of the detector to avoid any influence from the area on the MCP, where the detection efficiency is decreased since the incoming photons impinge on the MCP parallel to the MCP channels ${ }^{47}$ (cf. Methods, Scattering experiment). In order to fit the measured patterns for spherical particles, we employed a multidimensional Simplex optimization ${ }^{28}$ on the basis of multicolor Mie scattering calculations $\mathrm{s}^{29,30}$. The intensity pattern in a calculated multicolor image contains four single-frequency-components (11th to 17th harmonic), each weighted with the intensity of the respective harmonic order. As fitting parameters, the particle size and the photon energy-dependent refractive indices $(n=1-\delta+i \cdot \beta$ with $\delta$ being the deviation of the real part of the refractive index from unity and $\beta$, the imaginary part, which corresponds to the absorption) at the 13th and 15th harmonic were varied. For helium, the 11th and 17th harmonics are far away from resonances ${ }^{31,32}$, so that tabulated literature values of the refractive indices could be fixed within the fit procedure $(17.2 \mathrm{eV}: n=0.97+i 0.0 ; 26.6 \mathrm{eV}: n=0.9964+i 0.041)$ (values taken from the NIST data base http://physics.nist.gov/PhysRefData/FFast/ html/form.html). A set of 18 measured patterns with clear rings up to maximum scattering angle were fitted using a hybrid Monte-Carlo Simplex optimization algorithm for a large ensemble of trajectories (see Supplementary Figs. 5 and 6). Each fitting trajectory was initialized with random start parameters in a reasonable range for the corresponding optimization parameters $(R=300 \mathrm{~nm}$ to $R=600 \mathrm{~nm}$ $\delta=-0.3$ to $\delta=0.2, \beta=0$ to $\beta=0.07$ ), and subsequently improved via Simplex optimization. The scattering patterns for nonspherical shapes were calculated in the discrete-dipole-approximation as implemented in ref. 40 by the superposition of four single-color calculations and using the average optical parameters of the 13th and 15th harmonic determined in our study (solution with dominant 13th harmonic, $20.4 \mathrm{eV}: n=0.9252+i 0.0178 ; 23.5 \mathrm{eV}: n=1.2688+i 0.0417)$. The custom computer codes used are available on request from the authors. 
Data availability. All the data used in this study are available on request from the corresponding authors.

Received: 5 October 2016 Accepted: 19 June 2017

Published online: 08 September 2017

\section{References}

1. Chapman, H. \& Nugent, K. Coherent lensless X-ray imaging. Nat. Phot. 4, 833-839 (2010).

2. Seibert, M. et al. Single mimivirus particles intercepted and imaged with an X-ray laser. Nature 470, 78-81 (2011).

3. Loh, N. et al. Fractal morphology, imaging and mass spectrometry of single aerosol particles in flight. Nature 486, 513-517 (2012).

4. Bostedt, C. et al. Clusters in intense FLASH pulses: ultrafast ionization dynamics and electron emission studied with spectroscopic and scattering techniques. J. Phys. B 43, 194011 (2010).

5. Gomez, L. et al. Shapes and vorticities of superfluid helium nanodroplets. Science 345, 906-909 (2014).

6. Barke, I. et al. The 3D-architecture of individual free silver nanoparticles captured by X-ray scattering. Nat. Comm. 6, 6187 (2015).

7. Xu, R. et al. Single-shot three-dimensional structure determination of nanocrystals with femtosecond X-ray free-electron laser pulses. Nat. Comm. $\mathbf{5}$, 4061 (2014).

8. Raines, K. et al. Three-dimensional structure determination from a single view. Nature 463, 214-217 (2009).

9. Bostedt, C. et al. Ultrafast X-ray scattering of xenon nanoparticles: Imaging transient states of matter. Phys. Rev. Lett. 108, 093401 (2012).

10. Gorkhover, T. et al. Femtosecond and nanometre visualization of structural dynamics in superheated nanoparticles. Nat. Phot. 10, 93-97 (2016).

11. Miao, J., Ishikawa, T., Robinson, I. K. \& Murnane, M. M. Beyond crystallography: Diffractive imaging using coherent $\mathrm{x}$-ray light sources. Science 348, 530-535 (2015).

12. Hergott, J.-F. et al. Extreme-ultraviolet high-order harmonic pulses in the microjoule range. Phys. Rev. A 66, 021801 (2002).

13. Hädrich, S. et al. Generation of $\mu$ w level plateau harmonics at high repetition rate. Opt. Express. 19, 19374-19383 (2011).

14. Rudawski, P. et al. A high-flux high-order harmonic source. Rev. Sci. Inst $\mathbf{8 4}$, 073103 (2013)

15. Takahashi, E. J., Lan, P., Mücke, O., Nabekawa, Y. \& Midorikawa, K. Attosecond nonlinear optics using gigawatt-scale isolated attosecond pulses. Nat. Comm. 4, 2691 (2013).

16. Hong, K.-H. et al. Multi-mj, khz, $2.1 \mu \mathrm{m}$ optical parametric chirped-pulse amplifier and high-flux soft x-ray high-harmonic generation. Opt. Lett. 39, 3145-3148 (2014).

17. Popmintchev, D. et al. Ultraviolet surprise: Efficient soft x-ray high-harmonic generation in multiply ionized plasmas. Science 350, 1225-1231 (2015).

18. Miao, J., Ishikawa, T., Anderson, E. H. \& Hodgson, K. O. Phase retrieval of diffraction patterns from noncrystalline samples using the oversampling method. Phys. Rev. B 67, 174104 (2003).

19. Sandberg, R. L. et al. Lensless diffractive imaging using tabletop coherent high-harmonic soft-x-ray beams. Phys. Rev. Lett. 99, 098103 (2007).

20. Ravasio, A. et al. Single-shot diffractive imaging with a table-top femtosecond soft x-ray laser-harmonics source. Phys. Rev. Lett. 103, 028104 (2009).

21. Chen, B. et al. Multiple wavelength diffractive imaging. Phys. Rev. A 79, 023809 (2009).

22. Seaberg, M. D. et al. Ultrahigh $22 \mathrm{~nm}$ resolution coherent diffractive imaging using a desktop $13 \mathrm{~nm}$ high harmonic source. Opt. Express 19, 22470-22479 (2011).

23. Zürch, M. et al. Real-time and sub-wavelength ultrafast coherent diffraction imaging in the extreme ultraviolet. Sci. Rep. 4, 7356 (2014).

24. Schütte, B., Arbeiter, M., Fennel, T., Vrakking, M. J. J. \& Rouzée, A. Rare-gas clusters in intense extreme-ultraviolet pulses from a high-order harmonic source. Phys. Rev. Lett. 112, 073003 (2014).

25. Allaria, E. et al. Highly coherent and stable pulses from the FERMI seeded freeelectron laser in the extreme ultraviolet. Nat. Phot. 6, 699-704 (2012).

26. Frassetto, F. et al. High-throughput beamline for attosecond pulses based on toroidal mirrors with microfocusing capabilities. Rev. Sci. Inst. 85, 103115 (2014).

27. Krausz, F. \& Ivanov, M. Attosecond physics. Rev. Mod. Phys. 81, 163-234 (2009).

28. Lagarias, J. C., Reeds, J. A., Wright, M. H. \& Wright, P. E. Convergence properties of the nelder-mead simplex method in low dimensions. SIAM J. Optim. 9, 112-147 (1998).

29. Mie, G. Beiträge zur Optik trüber Medien, speziell kolloidaler Metallösungen. Ann. Phys. 25, 377-445 (1908).

30. Bohren, C. \& Huffman, D. Absorption and Scattering of Light By Small Particles (John Wiley, 1983).
31. Surko, C. M., Dick, G. J., Reif, F. \& Walker, W. C. Spectroscopic study of liquid helium in the vacuum ultraviolet. Phys. Rev. Lett. 23, 842-846 (1969).

32. Lucas, A. A., Vigneron, J. P., Donnelly, S. E. \& Rife, J. C. Theoretical interpretation of the vacuum ultraviolet reflectance of liquid helium and of the absorption spectra of helium microbubbles in aluminum. Phys. Rev. B 28, 2485-2496 (1983).

33. Joppien, M., Karnbach, R. \& Möller, T. Electronic excitations in liquid helium: The evolution from small clusters to large droplets. Phys. Rev. Lett. 71, 2654-2657 (1993).

34. Toennies, J. P. \& Vilesov, A. F. Superfluid helium droplets: A uniquely cold nanomatrix for molecules and molecular complexes. Angew. Chem. Int. Ed. $\mathbf{4 3}$ 2622-2648 (2004).

35. Gomez, L. et al. Supplemental material to: Shapes and vorticities of superfluid helium nanodroplets. Science 345, 906-909 (2014).

36. Brown, R. A. \& Scriven, L. E. The shape and stability of rotating liquid drops. Proc. R Soc. Lond. A Math. Phys. Eng. Sci. 371, 331-357 (1980).

37. Baldwin, K., Butler, R. \& Hill, R. Artificial tektites: an experimental technique for capturing the shapes of spinning drops. Sci. Rep. 5, 7660 (2015).

38. Ancilotto, F., Pi, M. \& Barranco, M. Vortex arrays in nanoscopic superfluid helium droplets. Phys. Rev. B 91, 100503 (2015).

39. Bernando, C. et al. Shapes of rotating superfluid helium nanodroplets. Phys. Rev. B 95, 064510 (2017).

40. Sander, K. et al. Influence of wavelength and pulse duration on single-shot $\mathrm{x}$-ray diffraction patterns from nonspherical nanoparticles. J. Phys. B At. Mol Opt. Phys. 48, 204004 (2015).

41. Cirmi, G. et al. Cut-off scaling of high-harmonic generation driven by a femtosecond visible optical parametric amplifier. J. Phys. B At., Mol. Opt. Phys 45, 205601 (2012).

42. Levin, Z. \& Cotton, W. in Aerosol Pollution Impact on Precipitation (eds Levin, Z. \& W.R., C.) (Springer, 2009).

43. Niikura, H. \& Corkum, P. Attosecond and angstrom science. Adv. At. Mol. Opt Phys. 54, 511-548 (2007).

44. Mauritsson, J. et al. Coherent electron scattering captured by an attosecond quantum stroboscope. Phys. Rev. Lett. 100, 073003 (2008).

45. Poletto, L. et al. Micro-focusing of attosecond pulses by grazing-incidence toroidal mirrors. Opt. Express 21, 13040-13051 (2013).

46. Even, U. The even-lavie valve as a source for high intensity supersonic beam. EPJ Tech. Instrum. 2, 1-22 (2015).

47. Fukuzawa, H. et al. Electron spectroscopy of rare-gas clusters irradiated by x-ray free-electron laser pulses from sacla. J. Phys. B 49, 034004 (2016).

48. Rupp, D. et al. Generation and structure of extremely large clusters in pulsed jets. J. Chem. Phys. 141, 044306 (2014).

49. Porod, G. Die Röntgenkleinwinkelstreuung von dichtgepackten kolloiden Systemen. Kolloid-Zeitschrift 124, 83-114 (1951).

50. Sorensen, C. \& Fischbach, D. Patterns in mie scattering. Opt. Commun. 173, 145-153 (2000)

51. Loh, N. D. et al. Sensing the wavefront of $\mathrm{x}$-ray free-electron lasers using aerosol spheres. Opt. Express 21, 12385-12394 (2013).

\section{Acknowledgements}

The authors kindly acknowledge Bernd Schütte's excellent work for the initial development of the HHG beamline. D.R. thanks Andrey Vilesov, Christoph Bostedt, Bernd von Issendorff and Joachim Ullrich for helpful and enlightening discussions. Excellent support has been provided by the TUB-IOAP workshop. This project has received funding from DFG (Grants No. MO 719/13-1 and /14-1), from BMBF (Grant No. 05K13KT2), and from the European Union's Horizon 2020 research and innovation programme under the Marie Skłodowska-Curie grant agreement No. 641789. Further, T.F. acknowledges computational resources provided by the North-German Supercomputing Alliance (HLRN) and financial support from the Deutsche Forschungsgemeinschaft via SFB652/3, a Heisenberg Fellowship (grant No.: FE 1120/4-1) and from BMBF (grant No.: 05K16HRB). F.C. and M.N. acknowledge funding from ERC grants STARLIGHT (grant No. 637756), and ELYCHE (grant No. 227355).

\section{Author contributions}

D.R. and Y.O. performed the feasibility studies in advance of the experiment. F.F., L.P. A.T., F.C., and M.N. developed the microfocusing optics setup and implemented it together with N.M. and A.R. The helium jet was set up by B.L., D.R. and M.S. set up the CDI detection system, and J.Z. developed the data acquisition system. N.M. and A.R. operated the HHG source, and D.R., M.S., B.L., N.M. and A.R. assembled and carried out the experiment. K.S., C.P., and T.F. developed and performed the scattering simulations. D.R., N.M., B.L., J.Z., K.S., C.P., and T.F. analyzed the data with input from all authors. The manuscript was discussed and written with input from all authors.

\section{Additional information}

Supplementary Information accompanies this paper at https://doi.org/10.1038/s41467017-00287-z. 
Competing interests: The authors declare no competing financial interests.

Reprints and permission information is available online at http://npg.nature.com/ reprintsandpermissions/

Publisher's note: Springer Nature remains neutral with regard to jurisdictional claims in published maps and institutional affiliations.

(c) (i) Open Access This article is licensed under a Creative Commons Attribution 4.0 International License, which permits use, sharing, adaptation, distribution and reproduction in any medium or format, as long as you give appropriate credit to the original author(s) and the source, provide a link to the Creative Commons license, and indicate if changes were made. The images or other third party material in this article are included in the article's Creative Commons license, unless indicated otherwise in a credit line to the material. If material is not included in the article's Creative Commons license and your intended use is not permitted by statutory regulation or exceeds the permitted use, you will need to obtain permission directly from the copyright holder. To view a copy of this license, visit http://creativecommons.org/ licenses/by/4.0/.

(C) The Author(s) 2017 